\title{
Indução da atividade cíclica ovariana pós-parto em vacas de corte submetidas à interrupção temporária do aleitamento associada ou não ao tratamento com norgestomet-estradiol
}

\author{
Ovarian ciclicity induction in beef cows submited to temporary weaning associated \\ or not with norgestomet-estradiol
}

\author{
João Batista Souza Borges ${ }^{1} \quad$ Ricardo Macedo Gregory ${ }^{2}$
}

\section{RESUMO}

\begin{abstract}
Este experimento teve como objetivo comparar os efeitos do desmame temporário (DT) por 72 horas associado ou não ao uso de Norgestomet (NOR) e estradiol na fertilidade do estro induzido e no periodo reprodutivo de vacas de corte acíclicas no pós-parto. O grupo DT $(n=38)$ era constituído por vacas que tiveram os terneiros separados por 72 horas. $O$ grupo NOR $(n=29)$ foi constituido por vacas que receberam (dia 0) um implante subcutâneo de Norgestomet e uma aplicação de Valerato de estradiol (5mg) e Norgestomet (3mg), intramuscular. No momento da retirada dos implantes (dia 9), os terneiros foram separados por 72 horas. $O$ intervalo desmame-estro foi significativamente mais curto $(P<0,05)$ no grupo NOR em relação ao grupo DT (50,1 e 86,4 horas, respectivamente). A taxa de indução de estros foi significativamente maior $(P<0,05)$ no grupo NOR $(75,8 \% x$ $26,3 \%)$, mas as taxas de ovulação (63\% x 60\%), de prenhez no primeiro estro $(36 \% \times 20 \%)$ bem como na temporada de reprodução $(51,7 \% \times 44,7 \%)$ não diferiram $(P>0,05)$ entre os grupos. O grupo NOR apresentou uma menor incidência $(P<0,05)$ de periodos curtos de atividade lutea $(27,2 \%) \mathrm{em}$ relação as vacas não tratadas $(40 \%)$. Os resultados demostraram que o tratamento de vacas de corte acíclicas com Norgestomet e estradiol determina um aumento na manifestação de estros após o desmame temporário por 72 horas, sem interferir nas taxas de prenhez do primeiro estro e da estação reprodutiva.
\end{abstract}

Palavras-chave: vaca de corte, desmame temporário, Norgestomet

\section{ABSTRACT}

The present study aims to compare the effects of removing the calf for 72 hours associated or not with Norgestomet (NOR) implant and estradiol upon estrus induction and fertility in postpartum aciclic beef cows. The temporary weaning (DT) group $(n=38)$ was separated from their calves for 72 hours The NOR group $(n=29)$ received on Day 0 a $3 \mathrm{mg}$ Norgestomet implant subcutaneously and $5 \mathrm{mg}$ of estradiol valerate, and $3 \mathrm{mg}$ of Norgestomet intramuscular. On Day 9 the implants were removed and the calves were separated for 72 hours. The interval from weaning to estrous was significantly shorter $(P<0.05)$ for cows treated with progestogens (50.1 hours) compared with calf removal group (86.4 hours). The estrous induction rate was significantly higher $(P<0.05)$ for Norgestomet and estradiol treated cows, but ovulation rate, first pregnancy rate and at the end of the breeding season were similar for both groups. The Norgestomet and estradiol treatment of aciclic beef cows improved the estrous incidence after 72 hours tempory weaning. The results demonstrate that the treatment of aciclic beef cows with Norgestomet and estradiol increases the induction of estrous after 72 hours of temporary weaning, without interfering with the pregnancy rates of first estrous and in the reproductive season.

Key words: beef cow, temporary weaning, Norgestomet

\section{INTRODUÇÃO}

A pecuária de corte no Brasil caracteriza-se como uma atividade de produtividade reduzida em conseqüência, entre outros fatores, do baixo desempenho reprodutivo dos rebanhos. Do ponto de vista econômico, para alcançar a máxima eficiência produtiva, as vacas de corte devem manter um intervalo entre os partos próximo de um ano. Para STAGG et al. (1998), o atraso na retomada da atividade ovariana pósparto em decorrência da nutrição deficiente associada

\footnotetext{
${ }^{1}$ Médico Veterinário, Doutor, Professor Adjunto, Departamento de Medicina Animal, Universidade Federal do Rio Grande do Sul (UFRGS), Av. Bento Gonçalves, 9090, Porto Alegre, RS, 91540-000. E-mail:joao.borges@ufrgs.br. Autor para correspondência.

${ }^{2}$ Médico Veterinário, Doutor, Professor Adjunto, Departamento de Medicina Animal, UFRGS.
} 
à amamentação permanente do terneiro é a principal causa de falha da nova concepção.

Após o parto, uma seqüência de alterações endócrinas deve ocorrer para que a atividade cíclica seja retomada na vaca de corte. Neste processo, a adequada liberação de gonadotrofinas é influenciada por fatores fisiológicos e patológicos associados à condição nutricional nos períodos pré e pós-parto. $\mathrm{O}$ aumento na liberação de hormônio folículoestimulante (FSH) inicia-se na primeira semana pósparto, determinando crescimento folicular seguido de atresia devido à insuficiente liberação de hormônio luteinizante (LH). Logo após o parto, os pulsos de LH apresentam baixa amplitude e freqüência, aproximadamente 1 a cada 4 horas, enquanto, para que ocorra a ovulação, são necessários pulsos com intervalos de 40-60 minutos para estimular a máxima produção de estradiol. Entre 15 e 30 dias pós-parto, a hipófise anterior restabelece a quantidade de LH que se apresentava reduzida ao parto devido à inibição de sua síntese causada pelos altos níveis de esteróides no final da gestação (YAVAS \& WALTON, 2000).

Outro fator que interfere na freqüência dos pulsos de LH no pós-parto de vacas de corte está ligado à inibição da ação dos neurônios responsáveis pela liberação de hormônio liberador de gonadotrofinas (GnRH) no hipotálamo. Os principais mecanismos envolvidos no bloqueio do centro gerador de pulsos de GnRH estão relacionados com os mediadores liberados em condições de nutrição deficiente ou pela amamentação e presença do terneiro. O efeito inibitório sobre a liberação de LH durante a amamentação está associado ao reconhecimento do terneiro através do olfato e da visão e não exclusivamente ao ato da sucção dos tetos (GAZAL et al., 1998).

O estabelecimento de aporte alimentar adequado no período pré e pós-parto (GOTTSCHALL \& LOBATO, 1996), o alcance de uma condição corporal ideal ao parto (KUNKLE et al., 1994), o desmame temporário por 48 a 72 horas (SHIVELY \& WILLIAMS, 1989) e o desmame definitivo aos 60 dias (CARTER et al., 1980) são as alternativas de manejo mais utilizadas para reduzir as falhas reprodutivas das vacas de corte.

A interrupção do estímulo da mamada associada ao afastamento temporário do terneiro possibilita o aumento dos pulsos de GnRH e LH podendo determinar a manifestação de estro e a ovulação (SHIVELY \& WILLIAMS, 1989). Segundo BEAL (2000), as taxas de indução de estro e prenhez variam de 19 a $32 \%$ e 14 a $18 \%$, respectivamente, com a utilização desta prática de manejo. Fatores como o tempo de interrupção do estímulo da mamada
(MALVEN et al., 1986; WILLIAMS, 1990), a condição corporal e o ganho de peso pós-parto (STAGG et al., 1998), bem como o intervalo parto-desmame temporário (VALLE \& EUCLIDES FILHO, 1997) estão diretamente relacionados com as taxas de prenhez alcançadas com a prática do desmame temporário.

Outra alternativa utilizada para desencadear a retomada da atividade cíclica no pósparto de vacas de corte é a aplicação de terapias hormonais. O uso de progestágenos associado ao estradiol para promover a indução e sincronização de estros e a ovulação em vacas de corte acíclicas têm sido recomendado para reduzir o intervalo partoconcepção (ODDE, 1990). O Norgestomet, uma potente progesterona sintética utilizada comercialmente em implantes auriculares de silicone para bovinos por período de 9 a 10 dias, estabelece uma concentração do progestágeno que permite a manutenção e maturação do folículo dominante e ovulação ao final do tratamento devido ao aumento da freqüência dos pulsos LH desencadeado (YAVAS \& WALTON 2000). De acordo com os estudos de SMITH et al. (1983) e VALLE \& EUCLIDES FILHO (1997), a taxa de manifestação de estros e de ovulação é maior em vacas de corte quando os terneiros são separados por 48 ou 72 horas ao final do tratamento com Norgestomet. As taxas de prenhez dos estros induzidos por este tratamento em vacas de corte acíclicas variam entre 20 e 50\% (SMITH \& VICENT, 1972; ODDE, 1990), sendo influenciadas, principalmente, por fatores como o intervalo parto-tratamento e a condição corporal (KUNKLE et al., 1994).

A fertilidade do primeiro estro pós-parto está relacionada a fatores como o desenvolvimento folicular, a viabilidade do óvulo e a atividade lútea, interferindo na concepção e desenvolvimento embrionário (BRUEL et al., 1993; INSKEEP, 2000). Nos casos em que o estro é induzido pelo tratamento com progestágeno-estradiol, uma seqüência de eventos fisiológicos envolvendo os centros de manifestação de comportamento do cérebro, o hipotálamo, a hipófise anterior e os ovários deve ocorrer para que resulte na ovulação e no desenvolvimento adequado do corpo lúteo. Segundo DAY \& BURKE (2000), estes tratamentos promovem elevadas taxas de manifestação de estro, mas apresentam uma grande variabilidade nas taxas de ovulação. A prevalência de estros induzidos em vacas acíclicas tratadas com progestágeno e estradiol após o parto que não resultam em ovulação, segundo FIKE et al. (1997) é de $10 \%$, podendo variar de 5 a $46 \%$ (BURKE et al., 2001), de acordo com gravidade da condição de aciclia. 
Segundo ZOLLERS (1993), durante a transição entre o estado de aciclia e a retomada da ovariana, o primeiro ciclo estral freqüentemente tem curta duração ( $<17$ dias), devido ao menor período de vida do corpo lúteo, inferior a 10 dias. MANN \& LAMMING (2000) postularam que a baixa concentração de estradiol que precede a primeira ovulação pode determinar o desencadeamento prematuro do mecanismo de luteólise devido à ausência de inibição da síntese de receptores de ocitocina que permitem a liberação precoce de prostaglandina F2 $\alpha$. Para RAMÍREZ-GODÍNEZ et al. (1981), o tratamento com Norgestomet em vacas de corte reduz a incidência de ciclos curtos após o primeiro estro pós-parto.

Este trabalho teve como objetivo avaliar a retomada da atividade ovariana, a fertilidade do estro induzido, o desempenho reprodutivo de vacas de corte acíclicas tratadas com Norgestomet e estradiol e/ou submetidas ao desmame temporário por 72 horas. Através da dosagem de progesterona sérica após os tratamentos, determinou-se a incidência de períodos curtos de atividade lútea.

\section{MATERIAL E MÉTODOS}

O experimento foi conduzido na Fazenda Rodeio do Rincão, localizada no município de Restinga Seca na região da Depressão Central do Estado do Rio Grande do Sul, durante os meses de setembro de 1999 a março de 2000. Foram utilizadas 67 vacas multíparas de corte com terneiros ao pé (cruzas Charolês x Nelore x Hereford) com idades variando entre 4 e 8 anos em aciclia diagnosticada através da palpação retal e de duas determinações da progesterona sérica abaixo de $1 \mathrm{ng} / \mathrm{ml}$ com intervalo de 7 dias. Os animais foram mantidos em campo nativo com uma carga animal de $320 \mathrm{~kg} / \mathrm{ha}$, em piquete próximo ao centro de manejo, durante o período experimental. As vacas foram avaliadas quanto à condição corporal, utilizando-se escores de 1 (magra) a 5 (gorda) de acordo com KUNKLE et al. (1994), e pesadas até 7 dias após o parto e no dia do início dos tratamentos para estabelecer a variação de peso neste período. Os animais foram divididos em 2 grupos constituídos ao acaso, de acordo com o tratamento realizado. O grupo desmame temporário (DT) foi constituído por 38 vacas que tiveram os terneiros apartados por 72 horas (Dia 0). Neste período, as vacas foram levadas para um campo distante do centro de manejo, enquanto os terneiros permaneceram na mangueira com água e pasto verde à disposição. O outro grupo era composto por 29 vacas (NOR) que receberam como tratamento hormonal uma injeção intramuscular de $3 \mathrm{mg}$ de Norgestomet e $5 \mathrm{mg}$ de valerato de estradiol e um implante auricular subcutâneo contendo 3mg de Norgestomet, por 9 dias (CrestarIntervet, Brasil). No dia da retirada do implante auricular, todas as vacas tiveram seus terneiros desmamados temporariamente, por 72 horas (Dia 0).

O controle dos estros sincronizados pelos tratamentos foi realizado pela manhã e tarde, por 2 horas, durante 5 dias a partir da separação dos terneiros. Para a cobertura das fêmeas, foram utilizados 10 touros com aptidão reprodutiva comprovada, por um período de 50 dias. Os diagnósticos de prenhez foram executados por palpação retal em duas ocasiões: $45 \pm 5$ dias após os estros sincronizados e 60 dias após a retirada dos touros.

As coletas de sangue para as dosagens de progesterona sérica foram realizadas nos dias -16, -9, $0,7,14,18$ e 22 (dia $0=$ início do desmame temporário e retirada do implante de Norgestomet). A determinação da progesterona sérica foi realizada por radioimunoensaio em fase sólida utilizando-se um kit comercial com anticorpos específicos para progesterona (Coat-A-Count, Diagnostic Products Corporation, Los Angeles, USA). A atividade lútea após os tratamentos foi caracterizada de acordo com FIKE et al. (1997).

1- Progesterona sérica abaixo de $0,5 \mathrm{ng} \cdot \mathrm{ml}^{-1}$ (nível basal) nos dias $-16,-9,0,7,14,18$ e 22 . Vacas permaneceram em aciclia.

2- Progesterona sérica basal nos dias -9 e 0 , elevação de, no mínimo, $0,5 \mathrm{ng} \cdot \mathrm{ml}^{-1}$ no dia 7 , permanecendo elevada nos dias 14, 18 e 22 . Formação e desenvolvimento de um corpo lúteo padrão até 4 dias após o dia 0 .

3- Progesterona sérica basal nos dias -9 e 0 , aumento de, no mínimo, $0,5 \mathrm{ng}^{\mathrm{ml}}{ }^{-1}$ no dia 7 ou 14 , queda para níveis basais no dia 18 ou 22. Formação e desenvolvimento de corpo lúteo com atividade lútea de curta duração ( $<10$ dias).

4- Progesterona sérica basal nos dias -9 e 0 , e 7 , elevação de $0,5 \mathrm{ng} \mathrm{x} \mathrm{ml}^{-1}$ no dia 14 , novo aumento de $0,5 \mathrm{ng} \cdot \mathrm{ml}^{-1}$ no dia 18 , permanecendo elevada no dia 22. Formação de corpo lúteo a partir de 4 dias depois do dia 0.

5- Progesterona sérica basal no dia -9, elevação de, no mínimo, 0,5 ng. $\mathrm{ml}^{-1}$ no dia 0 ou 3 . Ovulação ou período de meta-estro entre os dias -9 e 0 .

As taxas ovulação, de prenhez do estro sincronizado, do período reprodutivo e a manifestação de atividade lútea curta foram comparadas entre os grupos através do teste de qui-quadrado. Os pesos corporais, escores de condição corporal, ganhos de peso e intervalos parto-tratamento foram submetidos 
à análise de variância. Para a análise dos dados, foi utilizado o programa estatístico SAS, versão 6.12.

\section{RESULTADOS E DISCUSSÃO}

Os escores de condição corporal moderados $(2,57 \pm 0,2$ e 2,64 $\pm 0,2)$ e os ganhos de peso atingidos pósparto $(0,33 \pm 0,19$ e $0,38 \pm 0,22 \mathrm{~kg})$ das vacas dos grupos DT e NOR (Tabela1) são semelhantes aos relatados por GOTTSCHALL \& LOBATO (1996). Nestas condições, segundo os autores, o período de aciclia pós-parto pode exceder a 120 dias, determinando taxas de prenhez inferiores a $40 \%$ em vacas de corte com cria ao pé. Os ganhos de peso no período pós-parto observados neste experimento não foram suficientes para compensar a condição corporal moderada ao parto no restabelecimento da atividade ovariana pós-parto.

Com relação aos parâmetros reprodutivos (Tabela2), observou-se que a taxa de indução de estros apresentou uma diferença significativa $(\mathrm{P}<0,05)$ entre o grupo de vacas do grupo DT $(26,3 \%)$ e NOR $(75,8 \%)$. A menor resposta do grupo DT deve estar associada à baixa condição corporal das vacas e ao de quadro de aciclia grave. Neste caso, concordando com as observações de STAGG et al. (1998), a retirada do estímulo negativo representado pela amamentação e a presença do terneiro não foi suficiente para a retomada da atividade ovariana. De acordo com KUNKLE et al. (1994) e BEAL (2000), existe uma relação direta entre a condição corporal e a taxa de manifestação de estro em resposta à remoção temporária do terneiro. MALVEN et al. (1986) demonstraram que vacas com baixa condição corporal apresentam discreto aumento nos pulsos de LH a partir de 36 e 72 horas após a retirada do terneiro. Segundo WILLIAMS (1990), vacas que apresentam condição corporal moderada no pós-parto somente manifestam estro acompanhado de ovulação depois de 6 dias da remoção do terneiro. No entanto, quando associado ao tratamento com Norgestomet-estradiol, o desmame temporário por 72 horas é capaz de promover maiores índices de manifestação de estro, como relataram VALLE \& EUCLIDES FILHO (1997).

Também foi observado um menor intervalo $(\mathrm{P}<0,05)$ e uma maior sincronia entre a retirada do terneiro e o início do estro nas vacas que receberam tratamento hormonal $(50,1 \pm 18,0$ horas $) \mathrm{em}$ comparação com o grupo DT $(86,4 \pm 25,2$ horas), provavelmente devido ao controle da onda de crescimento folicular decorrente da aplicação do valerato de estradiol no dia -9 , como constataram DAY $\& \operatorname{BURKE}(2000)$.

$\mathrm{O}$ grupo NOR teve uma maior $(\mathrm{P}<0,05)$ taxa de ovulação $(48,2 \%)$ comparado com o grupo DT $(15,7 \%)$ quando se consideram todas as vacas tratadas. Esta diferença foi determinada pela maior eficiência na indução da retomada da atividade cíclica proporcionada pela hormonioterapia. Considerando apenas as vacas que apresentaram estro $(\mathrm{NOR}=22 \mathrm{e} \mathrm{DT}=$ $10)$, as taxas de ovulação foram semelhantes $(\mathrm{P}>0,05)$, 63,6 e $60 \%$, respectivamente. Como as vacas utilizadas neste experimento apresentavam em média intervalos parto-tratamento superiores a 90 dias, provavelmente ocorreram sucessivas ondas de crescimento folicular seguidas de atresia antes da indução do primeiro estro. Talvez por este motivo, as taxas de ovulação observadas tenham sido maiores do que a relatada por MURPHY et al. (1990) (11\%), que acompanharam através de ultrasonografia, o desenvolvimento de folículos dominantes no período de 7 a 15 pós-parto.

A taxa de prenhez no estro sincronizado das vacas $\operatorname{NOR}(36,3 \%)$ não apresentou diferença significativa $(\mathrm{P}>0,05)$, quando comparada com a de vacas do grupo DT (20\%). Taxas de prenhez variando entre 20 e $50 \%$ para o primeiro estro pós-parto provocado após os tratamentos com progestágenos

Tabela 1 - Peso corporal, escore de condição corporal, ganho de peso pós-parto e intervalo parto-tratamento de vacas de corte acíclicas tratadas com Norgestomet-estradiol (NOR) e submetidas ao desmame temporário (DT) por 72 horas.

\begin{tabular}{|c|c|c|c|c|}
\hline & \multicolumn{2}{|c|}{$\begin{array}{c}\text { DT } \\
(n=38)\end{array}$} & \multicolumn{2}{|c|}{$\begin{array}{l}\text { NOR } \\
(\mathrm{n}=29)\end{array}$} \\
\hline & Média & DP & Média & $\mathrm{DP}$ \\
\hline Peso corporal ao parto $(\mathrm{kg})$ & $366^{\mathrm{a}}$ & 39 & $384^{\mathrm{a}}$ & 39 \\
\hline Peso corporal tratamento $(\mathrm{kg})$ & $384^{\mathrm{a}}$ & 40 & $405^{\mathrm{b}}$ & 43 \\
\hline Condição corporal ao parto & $2,2^{\mathrm{a}}$ & 0,3 & $2,3^{\mathrm{a}}$ & 0,3 \\
\hline Condição corporal ao tratamento & $2,6^{\mathrm{a}}$ & 0,2 & $2,6^{\mathrm{a}}$ & 0,2 \\
\hline Ganho de peso parto-tratamento $(\mathrm{kg} / \mathrm{dia})$ & $0,33^{\mathrm{a}}$ & 0,19 & $0,38^{\mathrm{a}}$ & 0,22 \\
\hline Intervalo parto-tratamento (dias) & $98,8^{\mathrm{a}}$ & 24,6 & $103,6^{\mathrm{a}}$ & 25,8 \\
\hline
\end{tabular}

Letras diferentes nas linhas apresentam diferença significativa $(\mathrm{P}<0,05)$

Ciência Rural, v. 33, n. 6, nov-dez, 2003. 
Tabela 2 - Incidência de estro, ovulação, atividade lútea e parâmetros reprodutivos após o desmame temporário (DT) precedido ou não de tratamento com Norgestomet-estradiol (NOR).

\begin{tabular}{|c|c|c|}
\hline & DT $(n=38)$ & $\operatorname{NOR}(n=29)$ \\
\hline Taxa de manifestação de estro (\%) & $10 / 38(26,3)^{a}$ & $22 / 29(75,8)^{b}$ \\
\hline Intervalo desmame-estro (horas) & $86,4 \pm 25,2^{\mathrm{a}}$ & $50,1 \pm 18,0^{b}$ \\
\hline Taxa de ovulação de vacas em estro induzido (\%) & $6 / 10(60)^{a}$ & $14 / 22(63,6)^{\mathrm{a}}$ \\
\hline Taxa de ovulação de vacas tratadas $(\%)$ & $6 / 38(15,7)^{\mathrm{a}}$ & $14 / 29(48,2)^{b}$ \\
\hline Manifestação de atividade lútea curta (\%) & $4 / 10(40)^{a}$ & $6 / 22(27,2)^{b}$ \\
\hline Taxa de prenhez $1^{\circ}$ estro $(\%)$ & $2 / 10(20)^{a}$ & $8 / 22(36,4)^{a}$ \\
\hline Taxa de prenhez $1^{\circ}$ estro/tratados $(\%)$ & $2 / 38(5,2)^{\mathrm{a}}$ & $8 / 29(27,5)^{b}$ \\
\hline Taxa de prenhez período reprodutivo (\%) & $17 / 38(44,7)^{\mathrm{a}}$ & $15 / 29(51,7)^{a}$ \\
\hline
\end{tabular}

Letras diferentes nas linhas apresentam diferença significativa $(\mathrm{P}<0,05)$.

em vacas de corte acíclicas também foram relatadas por SMITH \& VICENT (1972) e ODDE (1990).

De acordo com as determinações de progesterona sérica, as vacas submetidas apenas ao desmame temporário que manifestaram estro apresentaram maior incidência $(40 \%)$ de períodos curtos de atividade lútea, comparando-se com a de vacas do grupo NOR $(27,2 \%)$, concordando com as observações de RAMÍREZ-GODÍNEZ et al. (1981). Segundo YAVAS \& WALTON (2000), a aplicação de progestágenos em vacas acíclicas determina um aumento na freqüência dos pulsos de LH que permite a manutenção do crescimento folicular, a ovulação e o subseqüente desenvolvimento adequado do corpo lúteo. O mecanismo pelo qual o tratamento com progestágenos prolonga a vida do corpo lúteo, de acordo com ZOLLERS et al., 1993, provavelmente esteja associado à supressão de receptores de ocitocina no endométrio, que estão envolvidos na liberação precoce de prostaglandina $\mathrm{F} 2 \alpha$ durante a retomada da ciclicidade em vacas de corte com cria ao pé.

Comparando-se o desempenho ao final do período reprodutivo, o grupo NOR apresentou maior uma taxa de prenhez $(51,7 \%)$ que o grupo DT $(44,7 \%)$, mas esta diferença não foi significativa $(\mathrm{P}>0,05)$. As taxas de prenhez semelhantes sugerem não haver diferença no efeito sobre o restabelecimento da atividade cíclica pela ação do progestágeno associado ao estradiol e pelo desmame temporário.

\section{CONCLUSÕES}

O uso isolado do desmame temporário por 72 horas em vacas de corte acíclicas com escore de condição corporal moderado ao parto é pouco eficaz para indução de estros e da concepção ao primeiro serviço. O tratamento com Norgestomet e estradiol associado ao desmame temporário por 72 horas é eficiente na indução do primeiro estro pós-parto, mas não aumenta as taxas de ovulação e os índices de prenhez em vacas de corte acíclicas. A utilização desta hormonioterapia precedendo o desmame temporário por 72 horas reduz a incidência de períodos curtos de atividade lútea após o estro induzido.

\section{REFERÊNCIAS BIBLIOGRÁFICAS}

BEAL, W.E. Estrus synchronization of cyclic and anestrous cows with Sincro-mate B. In: BEEF CATTLE SHORT COURSE, 49., 2000, Gainesville. Proceedings... Gainesville: University of Florida, 2000. p.31-35.

BRUEL, K.F. et al. Factors affecting fertility in the postpartum cow: role of the oocyte and follicle in conception rate. Biology of Reproduction, v.48, p.655-661, 1993.

BURKE, C.R. et al. Effects of maturity of potential ovulatory follicle on induction of estrous and ovulation in cattle with estradiol benzoate. Animal Reproduction Science, v.66, p.161174,2001

CARTER, M.L. et al. Effect of gonadotropin-releasing hormone and calf removal on pituitary-ovarian function and reproductive performance in postpartum beef cows. Journal of Animal Science, v.51, p.903910,1980

DAY, M.L.; BURKE, C.R. Management of follicular growth with progesterone and estradiol within progestin-based estrous synchrony systems. In: BEEF CATTLE SHORT COURSE, 49., 2000, Gainesville. Proceedings... Gainesville : University of Florida, 2000. p. 7-15.

FIKE, K.E. et al. Estrus and luteal function in suckled beef cows that were anestrous when treated with intravaginal device containing progesterone with or without subsequent injection of estradiol benzoate. Journal of Animal Science, v.75, p.2009-2015, 1997 .

Ciência Rural, v. 33, n. 6, nov-dez, 2003. 
GAZAL, O.S. et al. Gonadotropin-releasing hormone secretion into third-ventricule cerebrospinal fluid of cattle: correspondence with tonic and surge release of luteinizing hormone and its inhibition by suckling and neuropeptide Y. Biology of Reproduction, v.59, p.676-683, 1998.

GOTTSCHALL, C.; LOBATO, J.F.P. Desempenho reprodutivo de vacas de corte primíparas submetidas a três lotações em campo nativo. Revista da Sociedade Brasileira de Zootecnia, v.25, p.46-47, 1996.

INSKEEP, E.K. Factors that affect embryonic survival in the cow: application of technology to improve calf crop. In: BEEF CATTLE SHORT COURSE, 49., 2000, Gainesville. Proceedings... Gainesville : University of Florida, 2000. p.139-153.

KUNKLE, W.E.; SAND, R.S.; ERA, D.O. Effect of body condition on productivity in beef cattle. In: FIELDS, M.J.; SAND, R.S. (Eds). Factors affecting the calf crop. Gainesville : CRC, 1994. p.167-178.

MALVEN, P.V. et al. Relationship among concentrations of four opioid neuropeptides and luteinizing hormone-releasing hormone in neural tissues of beef cows following early weaning. Journal of Animal Science, v.62, p.723-733, 1986.

MANN, G.E.; LAMMING, G.E. The role of sub-optimal preovulatory oestradiol secretion in the aetiology of premature luteolysis during the short oestrous cycle in the cow. Animal Reproduction Science, v.64, p.171-180, 2000.

MURPHY, M.G.; BOLAND, M.P.; ROCHE, J.F. Pattern of follicular growth and resumption of ovarian activity in post-partum beef suckler cows. Journal of Reproduction \& Fertility, v.90, p.523-533, 1990.

ODDE, K.G. A review of synchronization of estrus in postpartum cattle. Journal of Animal Science, v.68, p.817-830, 1990.

RAMÍREZ-GODÍNEZ, J.A. et al. Reducing the incidence of short estrous cycles in beef cows with norgestomet. Theriogenology, v.15, p.613-623, 1981.

SHIVELY, T.E.; WILliAMS, G.L. Patterns of tonic luteinizing hormone release and ovulation frequency in suckled anestrous beef cows following varying intervals of temporary weaning. Domestic Animal Endocrinology, v.6, p.379387,1989 .

SMITH, Jr. L.E.; VICENT, K.C. Effects of early weaning and exogenous hormone treatment on bovine reproduction. Journal of Animal Science, v.35, p.1228-1232, 1972.

SMITH, M. F. et al. Pituitary and ovarian responses to gonadotropin releasing hormone, calf removal and progestogen in anestrous beef cows. Journal of Animal Science, v.57, p.418424, 1983.

STAGG, K. et al. Effect of calf isolation on follicular wave dynamics, gonadotropin and metabolic hormone changes and interval to first ovulation in beef cows fed either of two energy levels postpartum. Biology of Reproduction, v.59, p.777-783, 1998.

VALLE, E.R.; EUCLIDES FILHO, K. Efeito da separação temporária do bezerro, após a remoção do implante de norgestomet, na manifestação do cio. In: REUNIÃO DA SOCIEDADE BRASILEIRA DE ZOOTECNIA, 34., 1997, Juiz de Fora. Anais... Juiz de Fora : Sociedade Brasileira de Zootecnia, 1997. p.110113.

WILLIAMS, G.L. Suckling as a regulator of postpartum rebreeding in cattle: a review. Journal of Animal Science, v.68, p.831-852, 1990.

YAVAS, Y.; WALTON, J.S. Induction of ovulation in postpartum suckled beef cows: a review. Theriogenology, v.54, p.1-23, 2000.

ZOLLERS, W.G. et al. Concentration of progesterone and oxycitocin receptors in endometrium of postpartum cows expected to have short or normal luteal phases. Journal of Reproduction \& Fertility, v.97, p.329-337, 1993. 